05

\title{
Ультракороткие импульсы с широким спектром излучения в волоконных лазерах с пассивной синхронизацией мод
}

\author{
(C) А.К. Комаров ${ }^{1}$, К.П. Комаров ${ }^{1, \uparrow, ~ Л . М . ~ Ч ж а о ~}{ }^{2}$ \\ ${ }^{1}$ Институт автоматики и электрометрии Сибирского отделения РАН, \\ 630090 Новосибирск, Россия \\ ${ }^{2}$ Цзянсуская ключевая лаборатория передовых лазерных материалов и устройств, \\ Совместный инновационный центр передовых лазерных технологий и промышленных разработок, \\ Школа физики и электронной техники, Университет Цзянсу, \\ 221116 Сюйчжоу, Китай \\ ฯ e-mail: komarov@iae.nsk.su
}

Поступила в редакцию 23.09.2019 г.

В окончательной редакции 24.12.2019 г.

Принята к публикации 09.01.2020 г.

На основе численного моделирования и аналитических расчетов исследована генерация волоконных лазеров в режиме стационарных ультракоротких импульсов с предельно широким спектром излучения. Показано, что спектральная ширина генерируемых импульсов может в несколько раз превышать ширину полосы усиления лазерной активной среды.

Ключевые слова: волоконные лазеры, пассивная синхронизация мод, генерационная динамика, спектры импульсов.

DOI: $10.21883 /$ OS.2020.04.49201.272-19

\section{Введение}

Генераторы ультракоротких импульсов света широко используются в различных областях науки и техники, включая исследование быстропротекающих процессов, высокоскоростную передачу информации, прецизионную обработку материалов, мониторинг окружающей среды, навигационные системы и т.д. В настоящее время одним из ключевых направлений дальнейшего совершенствования этого типа генераторов связано с пассивной синхронизацией мод волоконных лазеров. Волоконные лазеры обладают рядом уникальных свойств, которые выгодно отличают их от других типов генераторов. Они компактны, надежны, практичны, удобны в эксплуатации, устойчивы к техническим возмущениям, демонстрируют богатое разнообразие генерационных режимов. Для волоконных лазеров с пассивной синхронизацией мод характерны различного типа гистерезисные явления, бистабильности и мультистабильности (бистабильность между режимом непрерывной волны и режимом пассивной синхронизации мод, мультистабильности, связанные с генерацией различного числа идентичных ультракоротких импульсов в лазерном резонаторе в зависимости от начальных условий, мультигистерезисные зависимости числа генерируемых импульсов от накачки и т. д.) [1-7].

В настоящее время разработка и совершенствование волоконных лазеров происходит по различным направлениям, включая создание новых усиливающих сред для генерации излучения в различных спектральных диапазонах, поиск новых способов реализации нелинейных потерь для формирования ультракоротких импульсов, поиск путей увеличения энергии и интенсивности фор- мируемых импульсов света, а также увеличения их спектральной ширины. В работах $[8,9]$ продемонстрирована генерация световых импульсов со спектральной шириной, превышающей ширину полосы усиления используемой волоконной усиливающей среды. Этот результат получен для лазера с пассивной синхронизацией мод, работающего в режиме шумовых импульсов. Механизм столь значительного спектрального уширения генерируемого излучения связан с керровской нелинейностью показателя преломления волоконной среды. В недавно опубликованной работе [10] продемонстрирована возможность значительного спектрального уширения световых импульсов, пропускаемых через волоконный усилитель. Для реализации такой схемы спектрального уширения необходимы генератор ультракоротких импульсов света и волоконный усилитель. Достигнутое спектральное уширение световых импульсов также значительно превышало ширину полосы усиления волоконного усилителя. Наши исследования направлены на изучение возможностей столь значительного спектрального уширения генерируемого излучения непосредственно в волоконном генераторе световых импульсов.

Целью настоящей работы является определение потенциальных возможностей достижения предельно широких спектров световых импульсов, формируемых при пассивной синхронизации мод волоконных лазеров, работающих в режиме стационарных импульсов. Полученные результаты также могут быть использованы для дальнейшего увеличения спектральной ширины генерируемых импульсов при реализации дополнительных возможностей, возникающих при работе лазера в режиме шумовых импульсов. В экспериментальной работе [9] в 
таком режиме генерации эрбиевого волоконного лазера была достигнута спектральная ширина генерируемого излучения в $203 \mathrm{~nm}$ при ширине полосы усиления, равной $25 \mathrm{~nm}$ [11]. Для продвижения в область более широких спектральных полос генерируемого излучения требуется соответствующая оптимизация параметров лазерной системы. Полученные в настоящей теоретической работе результаты для режима стационарных импульсов могут являться основой для обобщения разработанной теории на более сложный случай режима шумовых импульсов [9] с целью проведения такой оптимизации.

Статья структурирована следующим образом. В разд. 1 дан анализ спектров формируемых импульсов в простейшей модели лазерной генерации, допускающей аналитическое решение задачи. В разд. 2 представлена лазерная модель, более адекватно описывающая формирование ультракоротких импульсов света с предельно широким спектром излучения. В следующем разд. 3 представлены результаты формирования таких импульсов в рамках предложенной модели, учитывающей особенности их взаимодействия с усиливающей средой, а также дано обсуждение полученных результатов. В заключении приведены основные выводы по результатам выполненной работы.

\section{1. Генерационное уравнение с квадратичной дисперсией и кубической нелинейностью}

На рис. 1 приведена схема исследуемого волоконного лазера. Лазер включает в себя оптическое волокно, формирующее кольцевой резонатор, усилитель (Amplifier), насыщающийся поглотитель (SA), создающий нелинейные потери для формирования ультракоротких импульсов, и ответвитель для вывода части излучения из резонатора. Простейшая модель лазерной генерации с квадратичной частотной дисперсией и кубической нелинейностью внутрирезонаторной среды лазера допускает аналитическое решение поставленной задачи, которое может служить предварительной основой для дальнейшего уточнения теории. Соответствующее генерационное уравнение, описывающее лазер с распределенной внутрирезонаторной средой, имеет вид $[12,13]$

$$
\frac{\partial E}{\partial \xi}=\left(D_{r}+i D_{i}\right) \frac{\partial^{2} E}{\partial \tau^{2}}+\left[-\sigma+(p+i q)|E|^{2}\right] E,
$$

где $E$ - амплитуда напряженности поля, $\tau$ - временна́я переменная в единицах $\delta t=\sqrt{\left|\beta_{2}\right| L / 2}\left(\beta_{2}-\right.$ дисперсия второго порядка групповой скорости внутрирезонаторной среды, $L$ - длина резонатора), $\xi$ - пройденное излучением расстояние, нормированное на резонаторную длину $L$ (число проходов излучения через лазерный резонатор), $D_{r}$ и $D_{i}$ - частотные дисперсии усиления и показателя преломления волокна, $\sigma$ - линейные регенеративные потери, включающие линейные потери $\sigma_{0}$, связанные с выходом части излучения из резонатора,

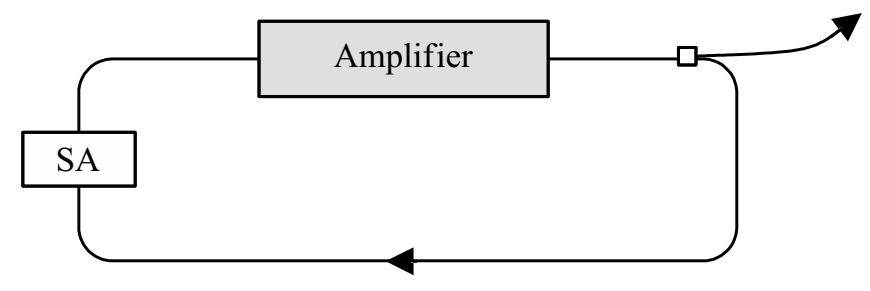

Рис. 1. Схема исследуемого лазера.

линейные потери $\sigma_{1}$, связанные с насыщающимся поглотителем, и усиление $g\left(\sigma=\sigma_{0}+\sigma_{1}-g\right)$. Усиление $g$ описывается [4] уравнением

$$
g=\frac{a}{1+b \int I d \tau}
$$

где $a$ и $b$ - параметры накачки и насыщения усиливающей среды, $I=|E|^{2}-$ интенсивность излучения, выраженная в единицах $(\gamma L)^{-1}$ (здесь $\gamma\left(\mathrm{W}^{-1} \mathrm{~m}^{-1}\right)-$ размерная нелинейность показателя преломления). Величины $p$ и $q$ - безразмерные нелинейности потерь и показателя преломления соответственно.

Описанная модель была разработана и использовалась ранее для анализа пассивной синхронизации мод как твердотельных, так и волоконных лазеров [12-15]. В рамках этой модели время релаксации нелинейных потерь и показателя преломления предполагается малым по сравнению с длительностью формируемых ультракоротких импульсов. Типичные длительности анализируемых импульсов лежат в пикосекундном диапазоне.

Стационарный импульс с независящими от $\xi$ распределениями интенсивности $I(\tau)$ и частотного чирпа ищем в виде

$$
E_{s}=\frac{E_{0} \exp (-i \Omega \xi)}{\cosh ^{1+i \alpha} \beta \tau},
$$

где $\Omega$ - нелинейно-дисперсионная поправка к несущей частоте импульса, $\beta$ - обратная длительность импульса, $\alpha$ - параметр частотного чирпа. Подставляя (3) в (1), получаем систему алгебраических уравнений на параметры импульса $\alpha, \beta, E_{0}[12]$ :

$$
\begin{gathered}
D_{i}\left(2-\alpha^{2}\right) \beta^{2}+3 D_{r} \alpha \beta^{2}=q\left|E_{0}\right|^{2}, \\
D_{r}\left(2-\alpha^{2}\right) \beta^{2}-3 D_{i} \alpha \beta^{2}=p\left|E_{0}\right|^{2} . \\
D_{r}\left(1-\alpha^{2}\right) \beta^{2}-2 D_{i} \alpha \beta^{2}=\sigma .
\end{gathered}
$$

Уравнения (4)-(6) совместно с (2) составляют полную систему уравнений, определяющих основные параметры импульса: $E_{0}, \beta, \alpha$ и усиление $g$.

Поделив уравнение (4) на (5), мы получаем уравнение, из которого находим значение частотного чирпа $\alpha$ через нелинейно-дисперсионные параметры задачи:

$$
\alpha=-\frac{3(1+\xi \theta)}{2(\xi-\theta)} \pm \sqrt{\left[\frac{3(1+\xi \theta)}{2(\xi-\theta)}\right]^{2}+2},
$$

где $\xi=q / p, \theta=D_{i} / D_{r}$. Знак в (7) выбирается из условия $\alpha>0$ при $\xi>\theta, \alpha<0$ при $\xi<\theta(\alpha=0$ при $\xi=\theta)$. 


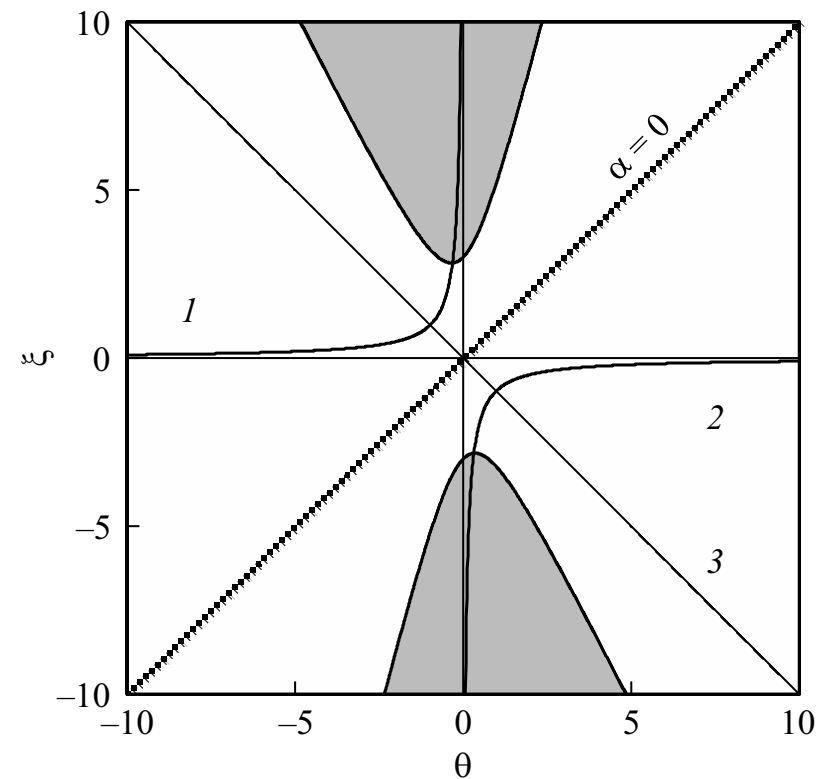

Рис. 2. Пунктирная прямая $\xi=\theta$ на плоскости $\xi, \theta$ соответствует спектрально-ограниченным импульсам $(\alpha=0)$. Для значений $\xi, \theta$, соответствующих гиперболе $1(\xi=-1 / \theta)$, частотный чирп $\alpha=\sqrt{2}$, для гиперболы $2(\xi=1 / \theta)-\alpha=-\sqrt{2}$. Прямая $3(\xi=-\theta)$ является осью симметрии, на которой располагаются вершины парабол, соответствующих кривым, вдоль которых частотный чирп $\alpha$ остается неизменным. Для нелинейно-дисперсионных параметров из затемненной области стационарный импульс является неустойчивым.

Характер изменения $\alpha$ при изменении параметров $\xi$, $\theta$ показан на рис. 2. При удалении от прямой $\xi=\theta$ в перпендикулярном направлении абсолютное значение частотного чирпа $|\alpha|$ увеличивается, и на кривых $\xi=-1 / \theta$ и $\xi=1 / \theta$ оно принимает значения $\sqrt{2}$ и $-\sqrt{2}$ соответственно.

Кривые на плоскости $\xi, \theta$, вдоль которых частотный чирп остается неизменным ( $\alpha=$ const), являются гиперболами, вершины которых располагаются на прямой $\xi=-\theta$ и направлены к началу координат $(\xi=0, \theta=0)$. Эта прямая является осью симметрии гипербол. Для заданного частотного чирпа $\alpha$ координата вершины соответствующей гиперболы на этой оси определяется выражением $\theta=\left(2-\alpha^{2}\right) / 3 \alpha \pm \sqrt{1+\left(\left(2-\alpha^{2}\right) / 3 \alpha\right)^{2}}$ (знак плюс берется для $\alpha<0$, знак минус - для $\alpha>0)$. Асимптотики для этой гиперболы определяются прямыми $\theta=\left(2-\alpha^{2}\right) / 3 \alpha, \xi=-\left(2-\alpha^{2}\right) / 3 \alpha$.

Изменение чирпа $\alpha$ вдоль оси симметрии описывается выражением (7) с $\xi=-\theta$. При больших значениях $|\xi| \gg 1,|\theta| \gg 1$ из (7) получаем

$$
\left.\alpha\right|_{\xi=-\theta} \approx-\frac{3 \theta}{2}=\frac{3 \xi}{2} .
$$

При удалении от оси симметрии в перпендикулярном направлении значение чирпа $|\alpha|$ уменьшается.

Прямая $\xi=\theta$ также является осью симметрии для любых двух гипербол с частотными чирпами, равными по абсолютной величине, но противоположными по знаку. Затемненная область на рис. 2 соответствует неустойчивости стационарного импульса, связанной с нарастанием поля вне импульса $(\sigma<0)$ [13].

Выражение (7) и одно из уравнений $(4),(5)$ позволяют определить параметры $E_{0}$ и $\beta$ через параметры задачи. Проведение исследования спектральных характеристик также возможно для фиксированного значения пиковой интенсивности $E_{0}$. При этом параметр накачки $a$ является подгоночным параметром, обеспечивающим заданное, фиксированное значение пиковой амплитуды импульса $E_{0}$.

Спектральная ширина излучения по полувысоте спектрального профиля для импульса в форме (3) определяется [12,13] выражением

$$
\Delta \omega=\frac{2 \beta}{\pi} \ln \left[(2+\cosh \pi \alpha)+\sqrt{(2+\cosh \pi \alpha)^{2}-1}\right] .
$$

При $\pi|\alpha| \ll 1$ получаем

$$
\Delta \omega \approx \frac{2 \beta}{\pi} \ln (3+2 \sqrt{2}) .
$$

При $\pi|\alpha| \gg 1$ (9) переходит в следующее выражение:

$$
\Delta \omega \approx 2|\alpha| \beta .
$$

После умножения (1) на комплексно сопряженную амплитуду напряженности поля $E^{*}$, прибавления к полученному выражению его комплексного сопряжения и интегрирования по временному интервалу локализации импульса получаем уравнение энергетического баланса для генерируемого импульса:

$$
\begin{aligned}
& \frac{d}{d \xi} \int|E|^{2} d \tau=-2 D_{r} \int\left|\frac{\partial E}{\partial \tau}\right|^{2} d \tau \\
& -2 \sigma \int|E|^{2} d \tau+2 p \int|E|^{4} d \tau .
\end{aligned}
$$

Для стационарного импульса (3) левая часть (12) равна нулю, и мы получаем соотношение между параметрами импульса при реализации такого баланса:

$$
2 p I_{0}=3 \sigma+D_{r} \beta^{2}+D_{r} \beta^{2} \alpha^{2} .
$$

Выигрыш в усилении $2 p I_{0}$, описываемый левой частью (13), связанный с насыщением нелинейных потерь, компенсирует регенеративные потери $\sigma$ и уменьшение усиления $D_{r} \beta^{2}$, обусловленное дисперсией усиления $D_{r}$ и конечной длительностью импульса $\beta^{-1}$, а также уменьшение усиления $D_{r} \beta^{2} \alpha^{2}$, связанное со спектральным чирпом импульса $\alpha$.

Ключевым параметром в достижении максимально широких спектров в режиме стационарного импульса является величина уменьшения нелинейных потерь $p I_{0}$ за счет их насыщения. Отметим, что в более точной теории, учитывающей нелинейности высоких порядков, 
изменение нелинейных потерь всегда меньше $\sigma_{1}$ (линейных потерь устройства, формирующего нелинейные потери). Для достижения максимальной ширины спектра, определяемой параметрами $\beta, \alpha \beta(10),(11)$, необходимо увеличивать диапазон изменения нелинейных потерь, описываемых в (13) слагаемым $p I_{0}$, и уменьшать линейные резонаторные потери $\sigma_{0}$, используя высокодобротные резонаторы. Соответственно из (13) с учетом (10) и (11) мы получаем следующее выражение для оценки достижения максимальной ширины генерируемого спектра:

$$
\Delta \omega \approx 2 \sqrt{\frac{2 \sigma_{1}}{D_{r}}} .
$$

При получении оценочного выражения (14) мы заменили в (13) изменение нелинейных потерь, описываемое слагаемым $p I_{0}$, на максимально возможное изменение нелинейных потерь $\sigma_{1}$, реализуемое при больших значениях накачки $a$.

Подставляя

выражение

для $D_{r}=(4 \ln 2) g /\left(\Delta \omega_{0}\right)^{2} \quad[16], \quad$ где $\Delta \omega_{0} \quad-$ ширина спектрального контура усиления по его полувысоте, получаем $\Delta \omega \approx \sqrt{2 / \ln 2} \sqrt{\sigma_{1} / g} \Delta \omega_{0}$. На границе устойчивости (рис. 2) $\sigma=0$ и соответственно $g=\sigma_{1}+\sigma_{0}$, следовательно, для достижения максимальной ширины генерируемого спектра, необходимо, чтобы линейные резонаторные потери $\sigma_{0}$ были малы по сравнению с линейными потерями устройства, создающего нелинейные потери, т.е. $\sigma_{0} \ll \sigma_{1}$, как это отмечалось выше. В этом случае $\Delta \omega \approx 1.7 \Delta \omega_{0}$. В случае иттербиевого волоконного лазера, описываемого рассматриваемой моделью (ширина полосы усиления $\Delta \omega_{0}=15 \mathrm{~nm}$ ) ширина спектра генерируемых импульсов может достигать $26 \mathrm{~nm}$. При этом, как следует из (11), длительность импульса, пропорциональная $\beta^{-1}$, будет нарастать по линейному закону с ростом параметра частотного чирпа $\alpha$, определяемого выражением (7). При $\alpha=0\left(D_{i}=0\right.$, $q=0)$ длительность формируемых импульсов может достигать $0.1 \mathrm{ps}$ (ширина спектра $10 \mathrm{~nm}$ ).

Режим чирпованных импульсов позволяет достичь тех же ширин спектра, что и спектрально-ограниченные импульсы, но при меньших значениях $\beta$, т.е. при больших длительностях импульса и соответственно при больших значениях его энергии.

\section{2. Модель генерации с гауссовым спектральным профилем усиления и насыщающимися потерями}

Когда ширина спектра становится сравнимой с шириной полосы усиления, то для анализа требуется более точная модель, чем модель с квадратичной дисперсией усиления, которая учитывала бы частотные дисперсии более высоких порядков. Кроме того, при высоких пиковых интенсивностях для описания нелинейных потерь требуется учет нелинейностей высоких порядков. Изменение поля в резонаторе за счет нелинейных элементов мы будем описывать [4] следующим уравнением:

$$
\frac{\partial E}{\partial \xi}=\left(-\sigma_{0}-\sigma_{n l}+i q|E|^{2}\right) E
$$

где нелинейные потери $\sigma_{n l}$ определяются выражением

$$
\sigma_{\mathrm{nl}}=\frac{\sigma_{1}}{1+p|E|^{2}} .
$$

Изменение спектральных компонент поля $E_{\omega}$, связанное с дисперсионными элементами, описывается уравнением

$$
\frac{\partial E_{\omega}}{\partial \xi}=\left(g F_{\omega}-i D_{i} \omega^{2}\right) E_{\omega},
$$

где спектральный профиль усиления $F_{\omega}$ выбирается гауссовым

$$
F_{\omega}=\exp \left(-D_{r} \omega^{2}\right) .
$$

Для такого профиля ширина полосы усиления $\Delta \omega_{0}$ по половине высоты $F_{\omega}(0) / 2$ определяется выражением $\Delta \omega_{0}=2 \sqrt{(\ln 2) / D_{r}}$. При ширине спектра генерируемого излучения, малой по сравнению с шириной полосы усиления $\left(D_{r} \omega^{2} \ll 1\right)$, выражение $(18)$ переходит в равенство $F_{\omega} \approx 1-D_{r} \omega^{2}$, и после фурье-преобразования уравнения (17) получаем следующее уравнение:

$$
\frac{\partial E}{\partial \xi}=\left(D_{r}+i D_{i}\right) \frac{\partial^{2} E}{\partial \tau^{2}}+g E,
$$

которое совместно с уравнением (15) в случае малых интенсивностей $\left(p|E|^{2} \ll 1\right)$ приводит к уравнению (1). Учет влияния спектрального контура $F_{\omega}$ на насыщение усиления приводит [17] к следующему уравнению для усиления:

$$
g=\frac{a}{1+(b / 2 \pi) \int F_{\omega}\left|E_{\omega}\right|^{2} d \omega} .
$$

В случае генерации узкополосного спектра $\left(F_{\omega} \approx 1\right)$ с учетом теоремы Парсеваля, из (20) получаем выражение (2).

Численное моделирование лазерной генерации проводилось на основе метода расщепления решаемой нелинейно-дисперсионной задачи на нелинейную и дисперсионную составляющие с использованием быстрого фурье-преобразования $[4,18]$. Начальное поле $E(\tau)$ бралось в форме гауссова импульса. На первом полушаге по $\xi$, используя выбранное начальное полевое распределение и уравнение (15), определяли эволюцию поля, связанную с нелинейными внутрирезонаторными элементами. Используя полученное распределение поля $E(\tau)$ как входное для уравнения (17), рассчитывали эволюцию поля, происходящую за счет дисперсионных элементов, реализуемую на втором полушаге по $\zeta$. Полученное распределение поля использовалось как входное для уравнения (15) для расчета эволюции поля на следующем шаге по $\xi$. Эта итерационная процедура повторялась до тех пор, пока не устанавливалось стационарное, не меняющееся от прохода к проходу 
через лазерный резонатор распределение излучения $(\partial I(\tau) / \partial \xi=0)$.

Предлагаемая модель позволяет оценить предельные спектральные ширины генерируемых световых импульсов, связанные с уширением спектра за счет нелинейности показателя преломления волоконной среды.

\section{3. Результаты численного моделирования и обсуждение}

Нелинейно-дисперсионные параметры волоконного лазера могут меняться в широких пределах. Полная нелинейность показателя преломления внутрирезонаторной среды может варьироваться за счет изменения длины волокна, формирующего лазерный резонатор. Полная частотная дисперсия показателя преломления может регулироваться за счет использования волокон с различной дисперсией, включая комбинацию волокон с частотной дисперсией показателя преломления противоположных знаков. Что касается увеличения ширины спектральной полосы усиления, то имеющиеся возможности здесь крайне ограничены и определяются существующим набором волоконных усиливающих сред. Численное моделирование проводилось с ориентацией на максимальное соответствие используемых параметров лазерной системы реально существующим характеристикам лазерных компонент.

Использовались следующие параметры генерационных уравнений, описанных в предыдущем разделе: $\sigma_{0}=0.1, \sigma_{1}=2.2, q=1.5, p=1, D_{i}=-1$ (нормальная дисперсия показателя преломления), $D_{r}=0.5, b=0.2$. Завышенное значение $\sigma_{1}$ бралось с целью достижения бо́льших спектральных ширин генерируемых импульсов. При численном моделировании мы также варьировали указанные параметры с целью определения зависимости генерационных характеристик от этих параметров.

Рисунок 3 демонстрирует возможность генерации стационарного импульса с шириной спектра $\Delta \omega$, существенно превышающей ширину спектральной полосы усиления $\Delta \omega_{0}$. Полученная ширина генерируемого спектра излучения $\Delta \omega$ в 3.7 раз больше ширины полосы усиления $\Delta \omega_{0}$ (при пиковой интенсивности импульса $\left.I_{0}=13.1\right)$. Этот результат находится в хорошем соответствии с аналитической оценкой, получаемой из выражения (14) и дающей отношение спектральных ширин, равное 1.7. Возникающее расхождение связано с тем, что квадратичная дисперсия приводит к более жесткой спектральной селекции излучения по сравнению с гауссовой моделью (18). Кроме того, значительное насыщение нелинейных потерь (параметр насыщения $p I \gg 1)$ также вносит свои коррективы в конечный результат. Длительность импульса, представленного на рис. 3, $a$, равна 4.2. При длине резонатора $10 \mathrm{~m}$ и параметре волоконной дисперсии $\beta_{2}=0.026 \mathrm{ps}^{2} / \mathrm{m}$ параметр $\delta t=0.36 \mathrm{ps}$, что дает размерную длительность импульса, равную $1.5 \mathrm{ps}$.
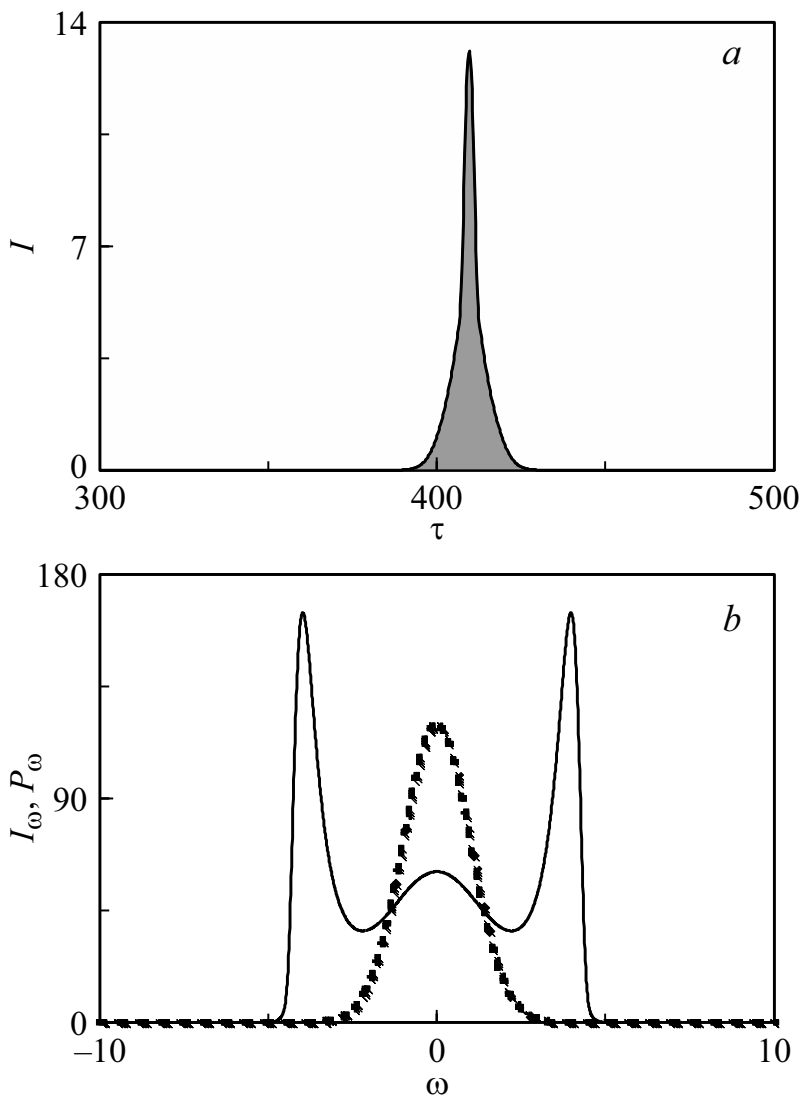

Рис. 3. (a) Временно́й профиль $I(\tau)$ стационарного импульса в устанавливающемся режиме генерации. (b) Спектральный профиль $I_{\omega}$ этого импульса (сплошная кривая). $P_{\omega}-$ спектральный профиль усиления (пунктирная кривая). $a=12$.

Возможность менять в широких пределах нелинейные потери, связанная с параметром $\sigma_{1}$, приводит к широкому интервалу накачек, для которых реализуется одноимпульсный режим с монотонным ростом ширины спектра генерируемого излучения при увеличении накачки $a$. Это демонстрируется на рис. 4, $a$. При $a=12.4$ из-за значительного спектрального уширения импульса эффективность его усиления падает, и новый импульс, имеющий меньшее спектральное уширение, возникает в генерации. В этом случае после переходного процесса реализуется режим генерации двух импульсов с идентичными характеристиками. Их энергии и пиковые интенсивности уменьшаются, и соответственно их спектральная ширина также уменьшается (рис. 4,a). В этом случае энергия накачки расходуется на генерацию двух импульсов, и соответственно характеристики каждого из импульсов будут примерно те же, что и в случае генерации одиночного импульса при накачке в два раза меньшей, т.е. при $a \approx 6.2$. Этим объясняется скачкообразное уменьшение интенсивности при достижении накачкой значения $a=12.4$. При реализации двухимпульсной генерации с ростом накачки (при $a=12.4$ ) и последующим уменьшении накачки мы наблюдали гистерезисную зависимость спектральной ширины $\Delta \omega / \Delta \omega_{0}$ от 

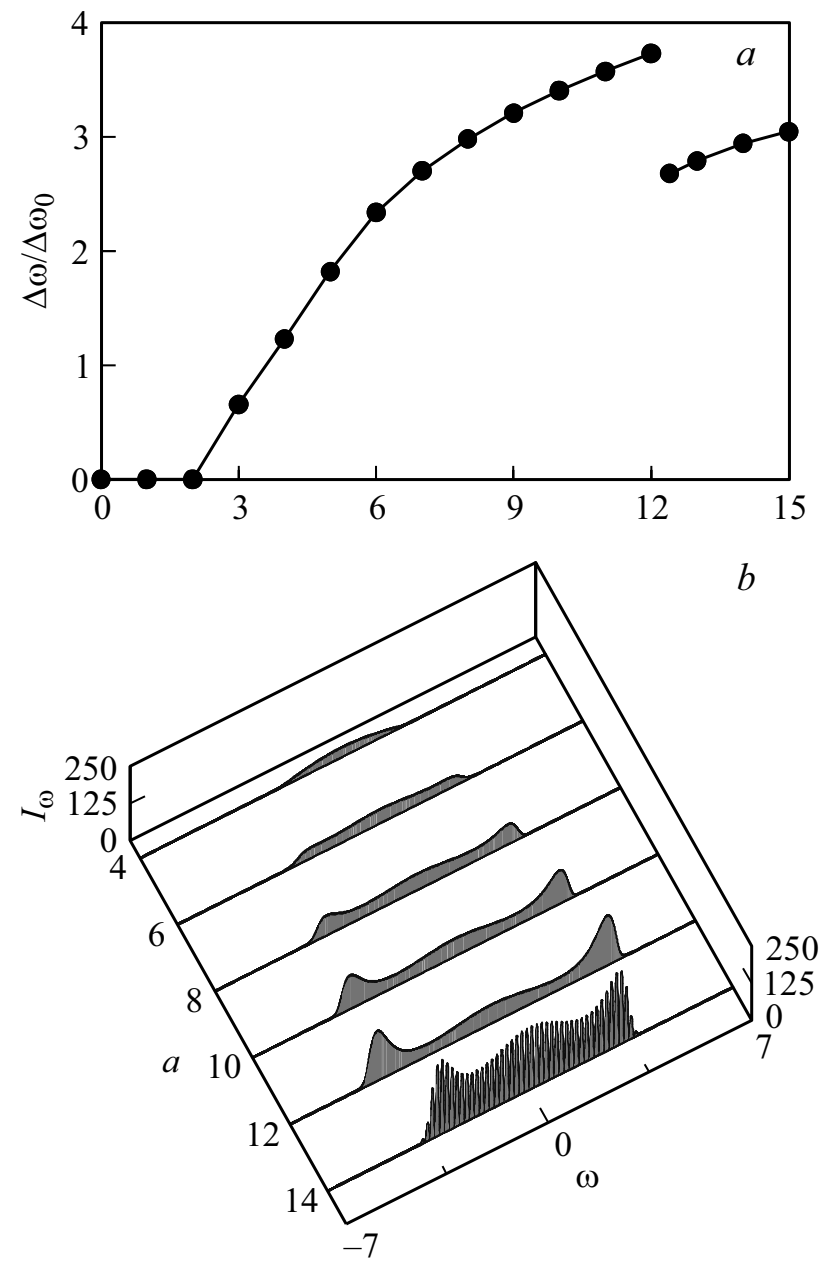

Pис. 4. (a) Отношение спектральной ширины генерируемого излучения к ширине полосы усиления $\Delta \omega / \Delta \omega_{0}$ как функция увеличивающейся накачки $a$. $(b)$ Изменение спектрального профиля генерируемого излучения $I_{\omega}$ при увеличении накачки $a$.

$a$ и соответственно генерационную бистабильность зависимость параметров излучения (ширины спектра, энергии излучения, количества импульсов (один или два) ит.д.) в устанавливающемся режиме генерации от начальных условий генерации. Эти генерационные особенности связаны с хорошо известной мультигистерезисной зависимостью числа идентичных диссипативных солитонов в устанавливающемся режиме генерации от накачки $[19,20]$.

Спектральный профиль двухимпульсного излучения (рис. $4, b$, частотная зависимость $I_{\omega}$ при $a=14$ ) оказывается промодулированным с частотой, равной обратному временному интервалу между этими импульсами. Бо́льшие значения параметра нелинейных потерь $\sigma_{1}(16)$ позволяет достичь бо́льших значений спектральных ширин генерируемых импульсов, реализуемых при увеличении накачки $a$.

Отметим, что с ростом накачки перед появлением в генерации второго импульса значение регенеративных потерь $\sigma=\sigma_{0}+\sigma_{1}-g$ приближается к нулю, что при- водит к формированию мощных импульсных крыльев (рис. 3,a). На этих крыльях концентрируется излучение, соответствующее высоким спектральным пикам, представленным на рис. $3, b$.

Рисунок 5 показывает изменение спектрального профиля при изменении параметра $\sigma_{1}$. Основные изменения связаны с увеличением числа генерируемых импульсов при уменьшении этого параметра, что приводит к перераспределению энергии между несколькими импульсами и соответственно к уменьшению ширины генерируемого спектра. Показанные на рисунке спектры при $\sigma_{1}=2.2,1.7$ соответствуют одноимпульсной генерации. При $\sigma_{1}=1.5,0.8$ реализуется двухимпульсный режим. Спектр излучения при $\sigma_{1}=0.6$ соответствует трехимпульсной генерации. То есть для достижения одноимпульсной генерации при высоких накачках $a$ требуется использование насыщающихся поглотителей с большими значениями параметра $\sigma_{1}$.

На рис. 6 показана плоскость параметров $q, D_{i}$ с областями устойчивой стационарной одноимпульсной и многоимпульсной генерации. При высоких пиковых интенсивностях формируемых импульсов, реализуемых при достаточно высоких накачках, происходит сильное насыщение нелинейных потерь, и соответственно неустойчивость одноимпульсной стационарной генерации возникает при меньших нелинейностях показателя преломления по сравнению со случаем кубической нелинейности внутрирезонаторной среды.

Механизм изменения числа импульсов в устанавливающемся режиме генерации при изменении параметров лазера и связь этой смены генерационного режима с границей устойчивости одноимпульсной генерации, показанной на рис. 2, ранее исследовались в работе [21].

Таким образом, для реализации ультракоротких импульсов с широким спектром излучения необходимы нелинейные потери с большой амплитудой модуляции, возникающей при изменении интенсивности от максимального внутрирезонаторного значения до нуля. В этом случае граница перехода к многоимпульсной генерации смещается в область бо́льших накачек. В результате в широких пределах реализуется одноимпульсная генерация, при которой спектральная ширина излучения монотонно увеличивается с ростом накачки. Появление в генерации при достаточно больших накачках новых импульсов не приводит к увеличению ширины спектра генерируемого излучения, хотя при этом растет мощность отдельных спектральных компонент. Мы ограничились исследованием режима стационарных импульсов. Перспективным направлением дальнейшего увеличения спектральной ширины генерируемых импульсов являются нестационарные режимы генерации, в частности режим шумовых импульсов $[8,9,22]$. Анализ предельных возможностей увеличения спектральной ширины генерируемого излучения для таких режимов является задачей дальнейших исследований.

Среди альтернативных подходов лазерной генерации световых импульсов со спектром, превышающим шири- 


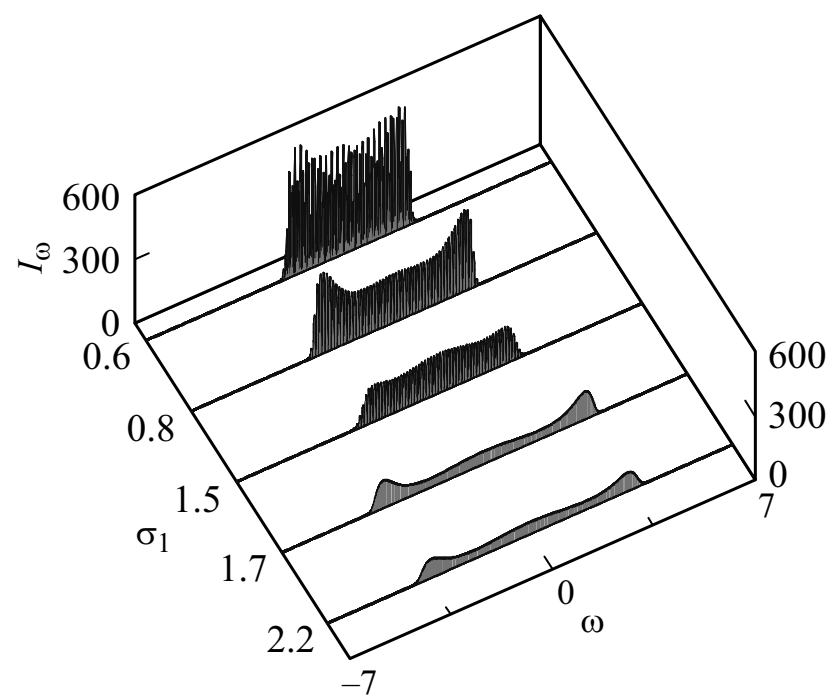

Рис. 5. Спектральный профиль стационарного импульса $I_{\omega}$ как функция параметра $\sigma_{1}$.

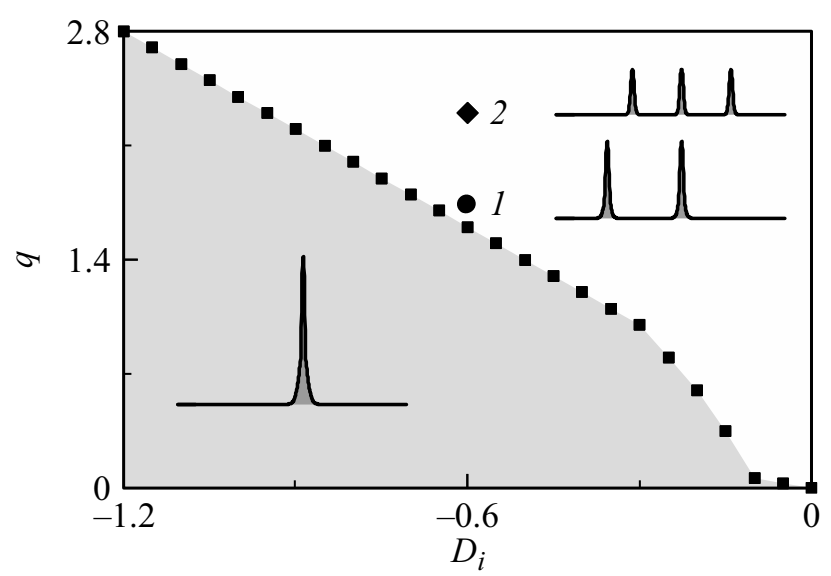

Рис. 6. Затемненная область параметров нелинейности $q$ и дисперсии $D_{i}$ показателя преломления соответствует устойчивой стационарной одноимпульсной генерации. Для параметров $q$ и $D_{i}$ из светлой области реализуется режим идентичных стационарных импульсов. Для параметров, соответствующих точке 1 , устанавливается двухимпульсная генерация. Для параметров точки 2 реализуется трехимпульсный режим.

ну спектральной полосы усиления-поглощения внутрирезонаторных сред, следует обратить внимание на механизм формирования импульсов, связанный с явлением самоиндуцированной прозрачности [23-28]. Хотя такой подход дает принципиальную возможность генерировать световые импульсы по длительности близкие к одному световому периоду [25], однако сама экспериментальная реализация такого режима наталкивается на значительные трудности. Тем не менее в недавней работе [23] такой режим генерации был экспериментально продемонстрирован. Для оценки конкурентноспособности такого подхода формирования импульсов по сравнению с традиционными потребуются значительные усилия для экспериментальной демонстрации его потенциальных преимуществ.

\section{Заключение}

В настоящей работе представлены результаты аналитических расчетов и численного моделирования формирования в волоконных лазерах с пассивной синхронизацией мод стационарных ультракоротких импульсов света с шириной спектра, сопоставимой и превышающей ширину полосы усиления активной среды. Механизм генерации излучения с такими спектральными полосами связан с керровской нелинейностью волокна, формирующего лазерный резонатор. Численное моделирование основывалось на предложенной модели, учитывающей особенности взаимодействия усиливающей среды и световых импульсов со столь широкими спектрами. Продемонстрирована возможность генерации световых импульсов с шириной спектра, в несколько раз превышающей ширину полосы усиления. Генерация таких импульсов реализуется при достаточно мощной накачке усиливающей среды и подавлении выхода в генерацию новых импульсов. Такое подавление связано с использованием насыщающихся поглотителей с высокой модуляцией нелинейных потерь при изменении интенсивности излучения от максимальных внутрирезонаторных значений до нуля. Полученные результаты являются основой для дальнейшего развития теории волоконных генераторов ультракоротких импульсов света с широким спектром излучения, работающих в нестационарных режимах, включая режим шумовых импульсов. Нестационарные режимы генерации обладают значительным потенциалом для дальнейшего увеличения спектральной ширины генерируемых импульсов. Такие генераторы имеют широкую область применений, включая различного типа спектроскопические исследования, когерентную томографию, локацию, мониторинг окружающей среды и другие приложения.

\section{Финансирование работы}

Исследование выполнено при финансовой поддержке Российского фонда фундаментальных исследований и Государственного фонда естественных наук Китая в рамках научных проектов № 19-52-53002, № 11911530083 и № 11674133 .

\section{Список литературы}

[1] Rosanov N.N. Spatial Hysteresis and Optical Patterns. Berlin, Heidelberg, NY: Springer-Verlag, 2002. 308 p.

[2] Tang D.Y., Man W.S., Tam H.Y. // Opt. Commun. 1999. V. 165. P. 189.

[3] Hideur A., Chartier T., Brunel M., Salhi M., Özkul C., Sanchez F. // Opt. Commun. 2001. V. 198. P. 141.

[4] Komarov A.K., Komarov K.P., Mitschke F.M. // Phys. Rev. A. 2002. V. 65. P. 053803. 
[5] Tang D.Y., Zhao L.M., Zhao B., Liu A.Q. // Phys. Rev. A. 2005. V. 72. P. 043816

[6] Grelu Ph., Akhmediev N. // Nature Photonics. 2012. V. 6. P. 84.

[7] Sanchez F., Grelu Ph., Leblond H., Komarov A., Komarov K., Salhi M., Niang A., Amrani F., Lecaplain C., Chouli S. // Opt. Fiber Technol. 2014. V. 20. P. 562.

[8] Zhao L.M., Tang D.Y., Cheng T.H., Tam H.Y., Lu C. // Opt. Comm. 2008. V. 281. P. 157.

[9] Wang X., Komarov A., Klimczak M., Su L., Tang D., Shen D., Li L., Zhao L. // Opt. Express. 2019. V. 27. P. 24147.

[10] Sidorenko P., Fu W., Wise F. // Optica, 2019. V. 6. P. 1328.

[11] Yang L., Zhu J., Qiao Z., Yan X., Wang Y. // Opt. Commun. 2010. V. 283. P. 3798.

[12] Комаров К.П. // Опт. и спектр. 1986. Т. 60. В. 2. С. 379; Komarov K.P. // Opt. Spectrosc. 1986. V. 60. № 2. P. 231.

[13] Комаров А.К., Комаров К.П., Дмитриев А.К. Нелинейная динамика формирования и взаимодействия ультракоротких импульсов в лазерах с пассивной синхронизацией мод. - Новосибирск: Изд-во НГТУ, 2017. 288 с.

[14] Komarov K.P., Kuch'yanov A.S., Ugozhayev V.D. // Opt. Commun. 1986. V. 57. N 4. P. 279.

[15] Комаров К.П., Кучьянов А.С., Угожсаев В.Д. // Автометрия. 1989. № 3. С. 108.

[16] Komarov A., Leblond H., Sanchez F. // Phys. Rev. A. 2005. V. 71. P. 053809.

[17] Ханин Я.И. Основы динамики лазеров. М.: Наука, Физматлит, 1999. $368 \mathrm{c}$.

[18] Taha T.R., Ablowitz M.J. // J. Comput. Phys. 1984. V. 55. P. 203.

[19] Komarov A.K., Komarov K.P. // Phys. Rev. E. 2000. V. 62. P. R7607.

[20] Sanchez F., Komarov A., Grelu P., Salhi M., Komarov K., Leblond $H$. // Nonlinear optical cavity dynamics: from microresonators to fiber lasers, Ed. by Grelu P. Wiley-VCH, Weinheim, 2016. Ch. 10. P. 231-262.

[21] Комаров А.К., Комаров К.П. // Опт. и спектр. 2003. Т. 94. № 1. C. 111; Komarov A.K., Komarov K.P. // Opt. Spectrosc. 2003. V. 94. N 1. P. 103.

[22] Komarov A., Komarov K., Zhao L. // Phys. Rev. A. 2019. V. 100. P. 033829.

[23] Архипов М.В., Архипов Р.М., Шимко А.А., Бабушкин И., Розанов Н.Н. // Письма в ЖЭТФ. 2019. Т. 109. С. 657.

[24] Arkhipov R.M., Arkhipov M.V., Babushkin I. // Opt. Comm. 2016. V. 361. P. 73.

[25] Arkhipov R.M., Arkhipov M.V., Babushkin I., Rosanov N.N. // Opt. Lett. 2016. V. 41. P. 737.

[26] Kozlov V.V., Rosanov N.N., Wabnitz S. // Phys. Rev. A. 2011. V. 84. P. 053810.

[27] Kozlov V.V. // Phys. Rev. A. 1997. V. 56. P. 1607.

[28] Комаров К.П., Угожсаев В.Д. // Квант. электрон. 1984. T. 11. № 6. C. 1167. 